\title{
Should I Stay or Should I Go? \\ A Study of Pickup Groups in Left 4 Dead 2
}

\author{
Jonas Linderoth \\ University of Gothenburg \\ Department of Education, Communication and Learning \\ jonas.linderoth@ped.gu.se
}

\section{Staffan Björk}

University of Gothenburg and Chalmers University

Department of Applied Information Technology staffan.bjork@ait.gu.edu

\section{Camilla Olsson}

University of Gothenburg

Department of Education, Communication and Learning camilla.olsson@ub.gu.se

\section{INTRODUCTION}

Trawling down memory lane, recalling the games that we used to play in childhood can be a bittersweet experience. For some of us memories of careless joy and happy days are blended with episodes of being excluded from play activities, chosen last to a team or leaving a game in a tantrum. Those who share such memories know that sometimes a game is not "just a game." Events that happen during an instance of play are affected by the shared history of the participants and can potentially shape future relations and identities. At the same time, we can recall how some games seemed to facilitate an exclusionary atmosphere while others did not seem to have this problem. The same child can be brutally excluded from a game of football only to hours later pass smoothly into a session of hide and seek. The nature of a game session is likely to be constituted between the identity of the partici- 
pants in the broader social context and the identity as a player in a rule governed game system. Who you are outside the game and who you become in the game will shape the social life of the encounter.

Today many play activities take place in digital online environments. Some of these online games enforce a very specific social structure: pickup groups (PUGs). PUGs are groups where the participants are randomly grouped together in teams and are expected to conduct collaborative tasks. Due to the virtual nature of these encounters the participants have no previous knowledge about whom they are collaborating with. The players are deprived of (or freed from) attributes like age, gender, ethnicity, class, social status and appearance as social resources (or stigma). They have no previous history with the other participants that can form a backdrop for their interactions. If the identities of the individual players are to somehow structure the activity they must be brought to life and accentuated by the participants through their actions, intentionally or not. From a historical perspective this is a new form of playground. In this article we investigate what it means for a play activity to take place in a social setting that for the participants is cut off from other settings outside the game session. What are the mechanisms for inclusion and exclusion in these situations? How are identities and social positions in and outside of the game session negotiated? What role does the particular design of the game play in structuring these activities?

In order to address these questions we have conducted an autoethnographic study of pickup groups (PUGs) in the game Left 4 Dead 2 (Valve 2009) (L4D2 hereafter). We have looked at how participants enter and leave these groups, how they negotiate their performances, present themselves, burst out in anger and make excuses. Our aim with the study was to contribute a deeper understanding of how these new social arenas are constituted by its' participants and the role game design plays in structuring these encounters. 


\section{Pickup groups}

PUGs fill the need for always having people to play together with in team-based games, even when no friends are available. Although PUGs in online games have existed since the earliest online First Person Shooters (FPS) such as Quake (id Software 1996) and Counter-Strike (Valve 1999), the types of demands on members of a PUG has evolved during recent years. The introduction of functional roles in Team Fortress (Valve 1999), Return to Castle Wolfenstein: Enemy Territory (Splash Damage 2003), the Battlefield (DICE 2002) series, and Defense of the Ancients (Eul 2003) has made it important that players maintain a suitable role composition for their team.

In the games mentioned above it is rare that any game information is passed between game sessions, and this can be put in contrast with games with persistent worlds, such as World of Warcraft (Blizzard 2004) and similar massively multiplayer online games (MMOGs). In these games the players can often, over time, build an online identity in the community on the servers where they play. This will mean that when doing a more collaborative task, such as an instance or group quest, their actions are related to their more stable identity on the server or in the community that they play. However as discussed by Eklund and Johansson (2010), even in games with persistent worlds there seems to be a recent trend towards making it easier to group people together by allowing teams to form over different servers, so-called 'cross realm instancing.'

PUGs constitute a new kind of social phenomenon. They are activity systems where players have to establish functional interaction patterns without using some of the social resources that are available to them in everyday life (Goffman 1961). In theory, such encounters are likely to display interaction patterns that facilitate communication and collaboration over social barriers. People who might not have collaborated in any other situation can find themselves working together. Typically, 
age is such an attribute that might be overcome as children and adults participate in the same game space (Linderoth and Olsson 2010).

\section{Two analytical strands}

Early accounts in the field of computer-mediated communication (CMC) expected that the anonymity of online communication would bring along boundary breaching social encounters. This idea turned out to be more complex since studies showed that the loss of a clear social identity rather leads to anti-normative behavior and in some cases to even stronger social categorizations (Postmes, Spears and Lea 1998). Later studies in this tradition have tried, on experimental grounds, to map the effects of anonymity, self-categorization and sense of belonging etc., as factors influencing communication and perception of others (see for example Carr, Vitak and McLaughlin 2013). While this research tradition might be applicable to the aspect of anonymity in PUGs it fails to provide analytical tools for dealing with the fact these meetings happen within games. To meet inside a game environment is not completely comparable to other forms of online encounters. PUGs are not only structured by social mechanisms they are also shaped by the materiality of the game's design. In order to understand the activity that emerges in a PUG one must study the interplay of social rules and game rules. That is the relation between social bonding in PUGs and the way these groups are designed into the game system.

Thus, in the study reported here, the gaming activity was interpreted with both concepts from micro-sociology as well as with concepts from the field of game design. In comparison to some of the early CMC studies, we also study online interactions outside of experimental settings. In our view, it is an empirical question if and how a certain feature in a game structures the gaming experience. This suggests that naturally occurring game activities would be the preferred unit of analysis. However, in order to understand if and how the materiality 
of a game system shapes the players' experience, interpretations of data should be made against an understanding of game mechanisms. Such an approach requires that the researcher supplement theories about their object of interest with knowledge about formal properties of games. In our case Goffman's ideas about the relation between a situated activity and the wider world $(1961,1986)$ is complemented with the gameplay design patterns approach (Björk and Holopainen 2005a; 2005b; Holopainen and Björk 2008).

\section{The game and the wider world - Goffman in game studies} Goffman's $(1961 ; 1986)$ theory has, in the literature on gaming, shown to be a powerful way of conceptualizing how games are local activity systems (Fine 1983; Hendricks 2006; Waskul 2006; Copier 2007; Calleja 2007; Consalvo 2009). According to this theory, we make sense of the world around us in accordance with the nature of the immediate situation at hand. Metaphorically, social episodes are seen as surrounded by a membrane (Goffman 1961) or a frame (Goffman 1986).

A framework is the more or less shared definition that participants in an activity have of the situation. It is the unspoken answer that participants give to the question: What is going on here? (Goffman 1961). The meaning of a spoken sentence, an object, an action or an event is dependent on which frame is currently established. Social encounters thus gain an organizational structure, i.e. rules that regulate who can participate, how labor is divided among participants, the position of leadership, and other social roles. Frames are something that the participants in an activity uphold and negotiate through their interaction. Analytically, one can observe how framing and negotiations of frames take place by paying close attention to how people talk and interact. If person A bumps into person B when walking on a sidewalk, we gain a lot of information about how person A "framed" the occurrence by observing the subsequent interaction (Goffman 1981). If person A re- 
sponds with "Oh I am so sorry" or "Look out you fool!" this will give us information about how A defined the situation.

The membrane or frame is constituted by transformation rules, i.e. social conventions for how things from the wider world affect the specific activity. To use Goffman's own example, if a chess player accidentally knocks over a chess piece over both players will make an effort in restoring the game board. Accidentally knocking over a game piece does not transform the situation at hand; the interaction membrane allows this event to occur without the game falling apart. The players thus take actions to make the event that occurred irrelevant (Goffman 1961). However, if a player were caught deliberately trying to hide one of the opponent's pieces while s/he looked away it would be another matter. The game would fall apart and the activity of accusation would arise.

The fact that participants uphold the social rules does not mean that any framework can emerge, or that social structures and discourses are of no importance. It is in the broader cultural context that participants find the resources for generating a certain activity:

"We cannot say the worlds are created on the spot, because, whether we refer to a game of cards or to teamwork during surgery, use is usually made of traditional equipment having a social history of its own in the wider society and a wide consensus of understanding regarding the meanings that are to be generated from it"

(Goffman 1961, 27-28)

According to Goffman, how encounters are organized depends significantly on how they are cut off from other potential forms of interaction: "An encounter provides a world for its participants, but the character and stability of this world is intimately related to its selective 
relationship to the wider one." $(1961,80)$.

\section{Gameplay design patterns meet Goffman}

In the essay Role Distance (1961) Goffman points out that mechanical operations can provide the basis for how an activity is structured. Goffman uses the example of a merry-go-round and discusses the roles of the operator, the merry-go-round riders and the audience looking at the activity. Each run of the merry-go-round becomes a distinguishable social unit, an instance that is structured by the materiality of the movement of the ride. Yet, this activity is organic in the sense that the participants can engage differently during the activity (1961, 96-99). Goffman illustrates this by pointing out that when the ride passes the audience, children can wave and smile to their parents but as soon as they are out of sight they get a bored expression on their face. The material structure of the ride blends with social rules and cultural conventions. In our view, this example illustrates a fruitful way of approaching the activity of playing. The materiality of the game technology and its built-in rules will structure the activity. Still, just as in the merry-go-round example, participants will not be victims to the system but able to shape their way of engaging with the game and other participants in the game. The activity will be constituted by both game mechanics and social mechanics.

Compared to a merry-go-round, the ways that a game will structure an activity is likely to be more complex and not always as evident. In our view the study of social life during gameplay has to take into account that the game has agency in structuring the interaction patterns that emerge. It is here that the game scholar needs to be knowledgeable in the field of game design and able to use concepts that describe system features of games as a part of her/his analysis. We used gameplay design patterns (Björk and Holopainen 2005a; 2005b; Holopainen and Björk 2008) as a conceptual framework in our study, specifically The gameplay design pattern collection (Björk 2012) that provides over 
300 detailed descriptions of different gameplay characteristics. These design patterns make it possible to analyze and see, how different rules interact or counteract and also provides a language for talking about system features in games (see also Zagal et al. 2005 for a similar approach to systematically describe game features). Gameplay design patterns can be used in many various ways, i.e. they do not constitute a dominant theory or method. In this work, they are primarily used to offer a more specific language to denote gameplay mechanics, which arguably are a vital part of the materiality of game design.

\section{ETHNOGRAPHY IN GAME ENVIRONMENTS}

The employed methodology in the study is a form of autoethnography. The authors have a vast experience of online games and have played the game L4D2 (as well as the first Left 4 Dead game) before the study was conducted. In this sense, the authors can be described as "complete-member researchers" (Ellis and Bochner 2000, 740). A difference between ethnography with participating observers and autoethnography lies in how to understand the involvement of the researcher and how to present the results. While some more traditional ethnographic accounts see a risk in the possibility that the researcher gets too emotionally involved in the field, i.e. "goes native" (for a discussion see Tedlock 2000, 455) autoethnography sees the emotions of the researcher, their subjectivity, as a resource to gain knowledge about the field (Ellis and Bochner 2000). Another difference is in how to present results, while traditional "fly on the wall" ethnography employs a passive voice, autoethnographies are, in some cases, autobiographies of the researchers (Ellis and Bochner 2000). In-game ethnography presupposes participating observers since it is hard to gain access to online game activities without taking part in them (see Mortensen 2002)—something that in our case, studying PUGs, was absolutely necessary. In our analysis, we have made use of our own subjectivity and experience but we use both passive voice and autobiographic accounts when presenting our results. This follows the 
approaches taken in game ethnographies on online games, e.g. Taylor's Play between worlds (2006) and Sundén and Sveningsson's Gender and sexuality in online game cultures (2012). A difference between these ethnographies and our study is that L4D2 does not have a persistent game world outside of each game session. Thus, with a focus on PUGs we did not establish any stable relations with the people we played with. On one hand this meant that the trust issues that can emerge in virtual ethnographies (cf. Hine 2008) was not an issue, on the other hand we could not complement our observations with interviews with those we played with.

Doing online studies also poses a challenge in terms of research ethics. In accordance with Thelwall (2010), we treated the online environment as a public space. PUGs are open to anyone who owns the game and there are no special invitations needed or password protections. People participate in short sessions with a nickname that they can change anytime and no history is recorded of the communication. This, and our main focus on the activity, made us conclude that as long as we protected the nicknames of the players there was no risk of any harm or violation of integrity. Yet it could be argued that we at least should have attempted to gain informed consent. Here it should be stressed that there is a large difference between studying a PUG in a team-based shooter game and a MMOG or MUD. In PUGs it is almost impossible to gain informed consent from the people who participate since a player can enter a game session and leave after some seconds and the phase of the activity does not allow longer chats. Eynon, Fry and Schroeder (2008) point to the possibility of identifying oneself as a researcher with one's online nickname. In our case that would have ruined our ability to see how elements from the wider world were negotiated into PUGs since we ourselves would have opened up for a very specific relation between the game session and a larger context. In the following, all nicknames have been changed and no information about game servers is provided. The informants are 
still complete strangers to the authors in almost all cases.

\section{Design and data collection}

During a period of eight months the authors did observations by participating in PUGs in L4D2 games and following forums discussing the game. The authors took written and recorded audio memos of their observations as well as logging chats and collecting screenshots. The quotes presented later are edited versions of these observations; this is partly due to clarity reasons and partly unavoidable since full notes could not be made while playing. In total, the authors spent approximately 200 play hours in the game with the aim of collecting data on PUGs. Yet, as complete-member researchers it is not obvious when research starts and stops, and the total amount of play hours that the researchers have spent in $L 4 D 2$ is significantly higher.

Nearly all observations were conducted in team vs. team mode, i.e. two teams competing against each other, on the Steam platform. The reason for choosing team vs. team as the primary mode is due to four factors. First, it is the mode where, in our experience, many players allocate a lot of their game time after becoming proficient at playing. Second, it allowed the study of more players at the same time, and inter-team communication. Third, playing against other humans typically puts greater demands on collaboration, and was thought more likely to provide clearer cases of excuses, negotiations of identity and vote kicking. Finally, in this mode the team takes turns being "infected." Playing on the infected side is different from being a survivor, which is the only team available in other game modes. Thus, versus mode offered us the possibility to do comparative observations regarding how game design structured the activity.

\section{PUGs in Left 4 Dead 2}

The following sections go through the various observations in our study, with an initial game description for those unfamiliar with the 
game. References to the most salient gameplay design patterns are made throughout the text, both as a way to stress observations about the gameplay design and to indicate how these were used as part of the research process. Since the study is based on the notes of three different researchers, we mention in the text before each excerpt which researcher it is who speaks.

\section{The game - basic design}

In $L 4 D 2$ four characters are grouped together into a team of survivors whose goal is to travel to safety in an apocalyptic world where nearly everyone else has become homicidal due to an infection. The basic mode of the game is a campaign-driven set of levels where players take the role of the survivors trying to reach safe rooms (a design pattern called Traverse, see Björk 2012) and gameplay typically swaps between players trying to use Stealth (Björk 2012) to avoid detection and Combat (Björk 2012) when this fails. At the start of the campaigns the players learn why they must again travel through the dangerous apocalyptic world, typically something has gone wrong with their rescue from a previous campaign, a helicopter has crashed, a car cannot drive on a blocked highway etc.

The other main mode in the game is team vs. team. Here one of the teams gets to take the roles of special infected that, together with the other infected, try to kill the survivors and stop them from reaching the safe room. This gives the teams Asymmetrical Goals (Björk 2012) but gameplay is further complicated by the fact that players on the "infected" team play as the special infected and have unique attacks (an example of Orthogonal Differentiation, players having different abilities, which also is found to a lesser degree in survivor teams due to having different weapons, see Björk 2012). To ensure balance this mode is played in a mirror fashion, i.e. first one team plays the survivors and the other the special infected, and the roles are reversed in the next round. Scores are dependent on how close the survivors get to the 
safe room, if they survive a level and actually get into the room they also gets a score bonus.

In the game, the support for collaboration and managing PUGs exist on many levels in the game design. To support the necessary coordination, players of both established groups (people that know each other from before) as well as PUGs typically make use of various forms of voice chat systems that are either built into the game, the gaming platform or third party systems such as Ventrilo, Skype, etc. Lobbies allow members of PUGs to introduce themselves to each other and discuss initial strategies. Voting systems allow group decisions on which level and difficulty to play, but also make it possible to kick people out of the game. Pre-recorded sound messages can quickly be sent to other players through a key press and mouse action, and these messages are in some cases context-sensitive to what the avatar is looking at.

\section{The 'brittle' frame - patterns for coming and going}

The dynamics of an activity is, according to Goffman $(1961 ; 1984)$, tied to the boundary of how the activity is sealed off from a wider context. Some elements emerging or entering the activity will be become integrated in the activity, other things will transform the activity and some things will destroy the activity making it fall apart. For example, an academic lecture can handle that members of the audience come and go but will break apart if there is a fire alarm. Someone bursting out in laughter at a funeral is a threat to the activity. While it might be handled as an awkward moment, the activity of collective sorrow might become transformed into a fight. A date will fall apart if one of the participants leaves the scene. Some games tend to have a rather rigid frame. It is dictated explicitly in the rules who is in the game and who is not, if a fan runs into a court where a sport takes place or a player is injured the game will make a pause. The unwelcome participant will have to be removed and the injured player looked after and sometimes substituted. The players will take actions that make these 
events irrelevant to the ongoing activity, putting brackets around the event, and in most cases it is possible to pick up the game again (Goffman 1961). There are some gameplay design patterns that explicitly govern how participants can enter and leave a game activity. In $L 4 D 2$, the Drop-In/Drop-Out pattern (Björk 2012) allows players to come and go without disturbing the game. Since the game also supports AI Players, i.e. the game adds AIs that take over characters when player leaves, the game is not as sensitive for dropouts as a MMO-instance. Players can come and go in both teams during team vs. team gameplay but if all players in one team leave then the server shuts down since human players are required in both teams. This design also makes it possible for players to drop into an ongoing campaign. This works smoothly in many cases and players come and go without this even being mentioned or noticed by the other participants sometimes. As Staffan experienced it is even possible to mistake a bot for another player:

I embarrassed myself again while playing tonight but I don't think anybody else noticed it. I had been playing for some time on a co-op server where people kept joining and leaving but the cooperation still worked well enough. So when I got pounced by a hunter and was rescued I typed a quick "thxs" before realizing it was a bot that helped me. It doesn't bother me that much if anybody else noticed since it's something most people do one time or another but it bothered me since one should keep track of who's a bot and who's not since they play differently.

As a social activity, L4D2 and other games with similar designs can be said to have unique properties in that they can handle participants coming and going. In terms of Goffman's (1961) membrane metaphor, these games are resilient to such a fundamental change as completely switching participants. It is hard to think of this happening offline in board meetings, dinner parties and other face-to-face activi- 
ties. Yet we can see this in team sports where players can be substituted in order to fill the position of forward, mid-fielder etc.

In some cases, we observed how the differences between human and AI players made the game pause. If one of the teams was not filled with human players in between chapters we observed how players employed a 'soft rule' (see Juul 2005) of waiting for that team to fill up. Generally, human players are considered superior to have in the team and thus it is seen as unfair if one team has fewer humans than the other team. Another reason for waiting for teams to fill up is that it minimizes the risk of having a whole team without human players, a game state that would immediately end the game in versus mode. As Jonas noted, this is a fragile balance.

Camilla and I were playing as infected and the other team, who were behind us with almost 200 points, lacked one player. When the chapter started they didn't leave the safe room but stood for minutes and waited for a fourth player. The other players in our team got frustrated by this downtime and urged them to start. One player in their team, Blinx234, agreed, and wanted them to start but the other two didn't go. Eventually the two other players in our team got bored and dropped out. Blinx234 said: Go before they all drop, and that made them start.

This example illustrates how players can negotiate the rules even in a digital game where technology upholds the rules. This negotiation of frame is done in relation to the risk of having the whole game fall apart, i.e. if everybody in the opposing team leaves.

The observations above show how the game activity in $L 4 D 2$ is stable, on the one hand, since it allows players to come and go. On the other hand, if people start to leave, the activity totally falls apart. Unlike many other social activities, there are no smooth transitions between 
different frames: you are either in the game or you are out of the game. The boundary between the game and the wider world can thus metaphorically be described as brittle. It is resilient to things that would transform and fundamentally alter other situated activity systems but once the game starts to fall apart the break is complete and there is no way to pick up the game state.

\section{Symbiotic Player Relations}

Looking at gameplay specifically, L4D2 encourages players to help each other in different ways. Infected can attack from many directions so watching different directions is often a good tactic, as is pointing out to others where weapons and tools are. However, player cooperation is primarily promoted through the attacks by the special infected - many of these incapacitate a survivor until the others have rescued him/her. This game thus utilizes the pattern of Helplessness (Björk 2012), a design were a player loses all form of agency over the game state (Bergström, Björk and Lundgren 2010). Tied to this Helplessness is also the pattern of Symbiotic Player Relations (Björk 2012). Players are, when playing on the side of the survivors, completely dependent upon each other, one player's performance in the game is in a very concrete manner tied to the performances of the rest of the team. In our experience, this gameplay pattern can create emotional pressure on the players. The phenomenon of rage, a player bursting out in anger, yelling and screaming and often dropping out from the game (rage-quitting) stems from situations of not being able to fully control one's own performance. An excerpt from Staffan's field diary illustrates this:

While playing the atrium finale of the Dead Center campaign we had yet another experience of playing with a player that thought he knew how to play and everyone that disagreed with him were idiots. When we didn't automatically follow his instructions he began ranting and then tried to vote kick the rest of the team. 
Disgusted he left the game but funnily enough he reappeared some minutes later on the opposing team. Apparently he soon concluded they were idiots as well since he soon tried to vote kick people there before leaving the game again. Needless to say, he didn't make the experience better for our team in either case.

A re-occurring observation is that players in versus mode matches often tend to explicitly blame teammates when their team is losing. Regardless of whether they are correct or not, there are many potential reasons for this: moving slowly, falling behind, rushing ahead, not helping teammates, or generally being unsuccessful. The language in the text chat typically becomes harsh when players start to blame other players. Posts on the official Left 4 Dead forum also ties the emotional pressure to this kind of game design and shows an awareness of the Symbiotic Player Relation in the game design. A player discussing rage quitting points out:

In co-op games, L4D especially, your success is tied SO CLOSELY to that of your teammates that invariably half the time you're [sic] going to lose simply because of unskilled teammates, which will naturally get people to leave. Point being that the "Rage quitter epidemic" shouldn't have really been much of a surprise to anybody. Sure wasn't to me.

Another player on the forums also expressed how the design of the game affects the atmosphere in the game. According to this player there is more annoying communication in L4D2 compared to the game Team Fortress 2:

-if someone annoys me in TF2, I can comfortably ignore them and focus on the other ten guys. Each obnoxious type is a quarter of the team and I'm going to rely on them closely to watch my back. Not worth it. 
In this study we saw how Symbiotic Player Relations was a design pattern that gave players a ground for negotiating their own identity as skilled or un-skilled players in relation to the performance of others. By negotiating who is and who is not fulfilling their role as a competent gamer the more sustainable identity that the players have outside the game is introduced and put at stake.

\section{Positions in a system - expectations and negotiations}

Goffman (1961) pointed out that in an activity there will be roles, specific identities tied to the expectations on the participants. In order for a lecture to happen, someone must take the role of a lecturer while others take on the role of the audience. A game like $L 4 D 2$ positions the participants as players. Jonas reflects upon this:

Again I found myself with players who did not use my nickname. When playing as an infected I was called "Boomer" or "Spitter" etc. depending on the kind of infected I was currently playing. One could expect this to be tied to the functionality of the different infected just like in an MMO instance where I have been addressed with my class "rogue go there, hunter trap the walker etc." However I have encountered this on the survivor side as well, being named as one of the characters Nick, Ellis, Coach or Rochelle. Always makes me feel so unimportant, just like I might as well be replaced by a bot.

Tied to this role of the player is the expectation that you are supposed to be skilled at playing the game. Even though players' opinions about what constitutes a skilled performance vary, the main reason we saw for trying to kick someone out was the perception that a certain player lacked skill. The presence of both Symbiotic Player Relations and Orthogonal Differentiation between players makes Role Fulfillment (Björk 2012) very important for both experiences of success and failure. To fail to protect teammates is something very visible; it is not like in 
other team-based games only affecting the score of the team, but also another players' agency.

Once gameplay has started two new roles will emerge: that of potential winners and that of potential losers. The score between the two teams becomes a structuring factor in what kind of roles the players can engage in. A lot of negotiation between the players is focused around these positions. Camilla writes in the field diary:

Tonight in one match we were behind the whole time. The other team was poor winners and provoked us to rage. I especially find it frustrating when they spam the laughter emote after you have failed an attack. The low score almost branded me and I wanted to communicate to the other players that I am not as bad as the score suggests. In the end I left quietly.

The urge to show the other participants that the performance here and now is not representative for who you are and your gaming skills, is a common theme in players' interactions. Immediately after an unsuccessful attack one can sometimes read the comment from the failing player simply saying: "lag", thus blaming the Internet connection for the event that just occurred. Other excuses are to blame bots on one's team or lay the blame on teammates. The accusation of other players being noobs (slang term for an unskilled, inexperienced player) is, according to our study, part of everyday interaction in L4D2. It is strategically used towards teammates in order to avoid having to take on the role of the loser. Here our observations seem to be in complete coherence with Goffman's theory:

"Often, during an encounter, a participant will sense that a discrepancy has arisen between the image of himself that is part of the official definition of the situation and the image of himself that seems to have just been expressed by minor untoward events 
in the interaction. He then senses that the participants in the encounter are having to suppress awareness of the new version of him, with consequent tension." (Goffman 1961, 51)

The unpleasant emotion of becoming stigmatized as a bad player is thus something that much of the interaction in L4D2 evolves around. Players tend to take precautions and interact in ways so that this can be avoided, something that can be done with very small means of communication.

\section{Communication patterns - a little information says a lot about who you are}

One striking thing about playing L4D2 is that although coordination is needed, there is often little communication during actual gameplay. This is due to the fast-paced nature of the game; writing longer pieces of text opens up opportunities for opponents to attack while one is defenseless.

Even though the game supports multiple communication channels (text messages, voice chats and predefined context sensitive comments accessible through a pie menu) in our experience this function is seldom used. There are many possible reasons for this, both technical and social in nature. Players may not have the proper technology like headsets or microphones, may not be quick typists or may not be familiar with the pie menu functionality.

An observation we made was that, even if the communication was sparse, it took rather little to affect the atmosphere of a game. A simple, "thanks" after rescuing someone, or "sorry" could emotionally mean a lot. When playing as survivors the interaction between the avatars is also a form of communication, i.e. one can assume something about other players from the way they are playing, if they wait for each other and share the resources one finds in the game. 
I noticed that I generally don't really like final battles; I tend to quit a game before they start. But sometimes I get this feeling for another player that can make me follow the game into the last level. Like when I played today and a player had given me a can of pills when I needed it. It just felt like I couldn't be the one who abandoned the team then. It felt like this player in a sense believed in me and I couldn't let him down by just dropping.

As Camilla notes here, it takes very small actions to gain a sense of another player. That is, by just being polite, players can establish a relationship that is not part of the game, a frame of sportsmanship. Sometimes politeness is used strategically in order to uphold the activity. When players have established a relationship that goes beyond the instrumental position they have to each other in team, they are more likely to feel committed to the game. We even saw a case where a player had invented a concept for trying to be polite so that people would stay; he called it "anti-quit talk." Players who excused themselves were not as likely to be kicked out either. Humor and self-criticism were also forms of communication that established relations between players that went beyond their position in the game system. In the excerpt above, the more stable attachment to the other player is what keeps Camilla in the game.

The fact that just a small amount of communication introduces the person behind the avatar is also something that can become a problem when players want to keep distance to other team members. In the excerpt below, Jonas deliberately avoids engaging with another player.

Again some young male voice tried to get verbal communication going in the voice chat. I did not answer. He tried to take a leadership role, telling other players what to do. He had a clear picture on how to play a certain level and thus played completely predictably. For me, part of the pleasure of the game comes from 
avoiding obvious strategies and being a bit creative in trying to outsmart the other team. On the first level in Swamp he went into the house where you always get caught and started to yell at me for passing it. Clearly I found his strategy inferior and thought that if I had gone inside we would have gotten even more stuck, giving the other team the possibility to spawn close. He kept on yelling in the voice chat so I turned off voice communication. Being silent makes it easy both to drop out and to suggest vote kicks. I don't think I have ever suggested a vote kick against anyone whom I have started to voice chat with.

As both the excerpts above suggest, it takes rather small means for players to establish a relation that is something 'more' than just positions in a game system. This relationship is, on the one hand, something that will give players a more solid relation and is thus an element that makes the 'blame game' less likely to emerge. On the other hand, as Jonas' excerpt shows, this more stable relation might be something that a player wants to avoid in order to be able to quit or vote kick. We also observed how stable relationships turned out to become an emotional dilemma: forming a stable in-game relation during a game session with someone, only to find out that when the wider world is introduced that the persons you play with have doubtful values.

\section{Misperception of others' identity - Dealing with racism, misogyny and homophobia}

As stated above, players in our study created more stable social activities by tuning down the importance of skill. If you have appreciated someone's joke you are more likely to show deference to this player's failures and low achievement. We found that this relief of pressure to perform made the game more fun (see Goffman 1961). Sharing a fun game with other players turned out to be one of the most boundary maintaining situations, something that in most cases was a positive experience. Yet, a stable framework could also cause problems when 
we found out that we had misperceived whom we were sharing the fun with. Jonas and Camilla had the following experience.

Tonight we found two other players speaking our language in the voice chat. They seemed really fun and we engaged in a conversation with them. None of us had played the level we played before but even though we did not find our way or good spots to attack, the other two players kindly instructed us. After an hour we added each other to our friend lists on Steam. At the final level, the climactic last fight before the rescue, everything was good and our team was winning. In a skilled move one of the other players was able to charge the black character Coach over the edge. When doing this he screamed out in the voice chat: "Did you see that Nigger fly!" and started to laugh. We felt gutted and wanted to leave but the commitment to the game was in this case so strong that we sat through it.

This experience was truly a surprise for these authors. The brittle, boundary-maintaining frame of the game kept the racist comment contained, i.e. it passed through the activity since the role-fulfillment was not threatened. As Goffman pointed out, some things will destroy the activity while others will pass through it. Playing in PUGs can thus sometimes mean that one has to deal with racism, misogyny and homophobia. While we in most cases left, the experience reported in the excerpt above shows how the brittle game frame also makes it possible to conserve problematic values. Jonas had a somewhat similar game experience:

Camilla and I were playing with another player who seemed pleasant. There was nothing special about him, I guess I understood him as nice due to the fact that he just made a couple of chat comments and that he didn't start blaming and accusing other players. After having played some levels with him, I saw a 
really offensive personal tag in a safe room [players can mark a safe room with a personal tag, a poster-like picture they upload to the game. Often these are explicit in nature]. The picture was a portrait of Hitler with the text "Tolerance is gay." I asked Camilla if she thought that it was our team mate who had posted it but learnt that it had been there when the other team was in the safe room. It must have been someone in their team who put it up there or it belonged to the server we played on. The moments while I was unsure about if it came from our team mate or not were really tense. Two thoughts went through my mind. First, it was an awakening about how extremely little I actually knew about this other person and how stupid it was of me to perceive him as "nice." Second, I imagined how awkward it would feel to kick someone who had nice manners after finding out he was a Nazi.

Again, the local identity of the player as someone that upholds functional game interaction, and values from the 'wider world' collided.

\section{DISCUSSION: GAME IDENTITIES AND THE WIDER WORLD}

Our study showed that when players communicate with each other, even if it is very sparse communication, they make a social agreement that seems to make kicking and rage quitting more unlikely. They present themselves as something more than just a position in the system. Goffman's metaphorical boundary between the game activity and the wider world thus becomes more solid, i.e. less likely to fall apart, when players show more of themselves than their local 'player identity.' Humor, self-criticism, politeness, etc. are social elements which give players a relationship outside the game-relevant domain and yet these observations indicate that they are crucial for the game experience. This means that elements like politeness can be strategically used in a meta-game where it is important to take measures so the activity 
does not fall apart, for instance through anti-quit talk.

These results point to the fact that even though PUGs are online activities, previous insights in the CMC field about flaming and anti-normative behavior might not always be easily transferable to game environments. Using Goffman (1961) as an interpretative framework make us interpret this as identity work of the players. The players put their identity at stake when they enter a PUG, who they can be will be tied to the skill they exhibit during a very short strip of interaction. Establishing a relation that goes beyond this meritocratic situation is a way of relieving the game session of the pressure of performing. By introducing the "wider world" in the game session the stakes are lowered. An unskilled performance will not create the same tension since the thing that just occurred is not representative of the whole person behind the avatar. From this perspective it is not so much the politeness as such that makes a game session seem friendlier. It is the fact that introducing the "wider world" in the game activity makes the activity become less skill-based since players become more considerate and start to show each other deference. Your local identity is not threatened by the fact that you made a mess of things since you can be something more than a bad player in the eyes of the other participants.

Since a game becomes more pleasurable if it is not on the verge of constantly falling apart, our observations partly explain why people tend to dislike PUGs and prefer to play with friends. Having a stable relation outside the game context relieves the players from the pressure of performing. It makes gaming less of a skill-based activity where we tend to show deference to the unskilled player-after all she or he is funny, nice, polite etc. It is here we find one function of guilds and clans in gaming. This conclusion would thus explain the socio-psychological mechanics of why online gamers prefer to play with friends rather than in PUGs (see Eklund \& Johansson 2010). 
From a design perspective this suggests that it is important to give PUG-players an ability to negotiate their identity when starting to loose, especially when the pattern of Symbiotic Player Relations is employed. In a game with leveling and statistics tied to the avatar there is always a possibility to blame uneven conditions as the main reason for losing. In a game with static avatars, like $L 4 D 2$, it might be an idea to give players some way out of the position of the loser, some design that makes it possible to blame conditions outside one's own agency.

The results discussed above have to do with how positive perceptions of other player's identities in the "wider world" comes to life in game sessions and sometimes can save a session that would otherwise fall apart. Our study also showed a somewhat reversed version of this structure. Negative perceptions of other player's out-of-game identities were not discussed as long as it did not disturb the pattern of Role-fulfillment. While humor and generosity could save a bad game from breaking, racism and misogyny did not necessarily make a stable game vulnerable. These observations suggest a somewhat rough formula to the understanding of the social mechanisms for gameplay; positive views of other players out-of-game identity can save a problematic game session from falling apart, negative views of other players outof-game identity does not make a smooth game session vulnerable or make it fall apart. While there might be an aftermath, such as players blocking each other or not playing again with the same players, the on-going game will not break. Clearly this formula will have to be investigated further and given the different social statuses games have in the wider world, one can expect large variations. Even a high stake football match can be subject to this structure. When soccer-player Giorgos Katidis in March 2013 took off his shirt and made a Nazi-salute to celebrate a goal, he was later banned for life from the Greek national team. Yet the on-going game was played to the end and the referee's booking had to do with the violation of taking off the shirt during gameplay, not the display of unacceptable values (101 great 
goals 2013). If games in general, and especially PUGs are social arenas where exhibitions of misogyny, racism, homophobia and other forms of intolerance are more likely to 'pass' than they would in other activities, then this is a worthy topic for further research. Some autoethnographic accounts in this study suggest that this might be the case.

\section{ACKNOWLEDGEMENTS}

The work presented here was sponsored by the Swedish Knowledge Foundation (project GRO) and was also a part of the research conducted at the The Linnaeus Centre for Research on Learning, Interaction and Mediated Communication in Contemporary Society (LinCS) funded by the Swedish Research Council.

The research was also funded by the Integrated Project TA2, Together Anytime, Together Anywhere. TA2 receives funding from the European Commission under the EU's Seventh Framework Programme. The authors gratefully acknowledge the financial support and the productive collaboration with the consortium partners.

\section{REFERENCES}

"101 great goals." Giorgos Katidis (AEK Athens) scores winner $v$ Veria, celebrates with Nazi Salute. 16 March 2013. Available at http:// www.101greatgoals.com/blog/giorgos-katidis-aek-athens-scoreswinner-v-veria-celebrates-with-nazi-salute/ (accessed April 2013)

Bergström, K., S. Björk, and S. Lundgren. "Exploring aesthetical gameplay design patterns: Camaraderie in four games," paper presented at the MindTrek 2010 Conference, 6-8 October 2010, Tampere, Finland.

Björk, S. Gameplay design patterns collection: Main page. 2012. Available at http://gdp2.tii.se/index.php/Main_Page (accessed April 2013).

Björk, S. and J. Holopainen. Patterns in game design. Charles River Media: Hingham, MA, 2005a. 
Björk, S. and J. Holopainen. "Games and design patterns", in K. Salen and E. Zimmerman (eds.), The Game Design Reader: A Rules of Play Anthology. MIT Press: Cambridge, MA, 2005b

Blizzard. World of Warcraft [PC]. Blizzard: San Francisco, CA, 2004. Calleja, G. "Digital game involvement: A conceptual model." In Games and Culture vol. 2 no. 3, 236-260, 2008.

Carr, C. T., J. Vitak, and C. McLaughlin. "Strength of social cues in online impression formation: Expanding SIDE research," in Communication Research vol. 40 no. 2, pp. 261-281, 2013.

Consalvo, M. "There is no magic circle," in Games and Culture vol. 4 no. 4, 408-417, 2009.

Copier, M. Beyond the magic circle: A network perspective on role-play in online games. Unpublished Ph.D., Utrecht University: Utrecht, the Netherlands. 2007.

Eklund, L., and M. Johansson. "Social play? A study of social interaction in temporary group formation (PUG) in World of Warcraft." Paper presented at Nordic DiGRA, Stockholm, Sweden, 2010.

Ellis, C. and A.P. Bochner. "Autoethnography, personal narrative, reflexivity: researcher as subject," in N. K. Denzin and Y. S. Lincoln (eds.), Handbook of qualitative research (pp. 733-768). Sage: Thousand Oaks, CA, 2007.

Eul. Defenders of the Ancients. Modification of Blizzard (developer) Warcraft 3. [PC] San Francisco, CA, 2003.

Eynon, R., J. Fry, and R. Schroeder. "The ethics of internet research," in N. Fielding, R. M. Lee and G. Blank (eds.), The handbook of online research methods (pp. 23-41). SAGE Publications: London, U.K., 2008.

Fine, G. A. Shared fantasy: Role-playing games as social worlds. Univ. of Chicago Press: Chicago, IL, 1983.

Goffman, E. Encounters: two studies in the sociology of interaction. Bobbs-Merrill: Indianapolis, IN, 1961.

Goffman, E. Forms of talk. University of Pennsylvania Press: Pennsylvania, 1981 . 
Goffman, E. Frame analysis: an essay on the organization of experience. Northeastern University Press: Boston, MA, 1986.

Hendricks, S. Q. "Incorporative discourse strategies in tabletop fantasy role-playing gaming," in P. J. Williams, S. Q. Hendricks, and K. W. Winkler (eds.), Gaming as culture: Essays on reality, identity, and experience in fantasy games. McFarland \& Company: North Carolina, 2006.

Hine, C. "Virtual ethnography: Modes, varieties, affordances," in N. Fielding, R. M. Lee, and G. Blank (eds.), The Sage Handbook of Online Research Methods pp. 257-270. SAGE: London, U.K., 2008.

Holopainen, J. and S. Björk. "Gameplay design patterns for motivation," in Proceedings of ISAGA 2008, Kaunas, Lithuania, July 2008.

id Software. Quake [PC] Activision: Los Angeles, CA, 1996. Juul, J. Half-real: Video games between real rules and fictional worlds. MIT Press: Cambridge, MA, 2005.

Linderoth, J. "The struggle for immersion: Narrative re-framings in World of Warcraft," in Proceeding of the The [Player] Conference, IT University of Copenhagen: Copenhagen, Denmark, 2008. Linderoth, J., and U. Bennerstedt. Living in World of Warcraft: The thoughts and experiences of ten young people. Medierådet: Stockholm, Sweden, 2007.

Linderoth, J., and C. Olsson. Världen som spelplan: Gränsöverskridande i onlinespelskulturen. Medierådet: Stockholm, Sweden 2011.

Mortensen, T. "Playing with players: Potential methodologies for MUDs" In Game Studies vol. 2 no. 1, 2002. Available at http:// www.gamestudies.org/0102/mortensen/ (accessed December 2013).

Murray, J. H. "The last word on ludology v. narratology in game studies." Presented at the International DiGRA Conference, Vancouver, Canada, June 2005.

Postmes, T., R. Spears, and M. Lea. "Breaching or building social 
boundaries? SIDE-effects of computer-mediated communication," in Communication Research 25, 689-715, 1998.

Splash Damage. Return to Castle Wolfenstein: Enemy Territory [PC]

Activision: Santa Monica, CA, 2003.

Sundén, J. and M. Sveningsson. Gender and sexuality in online game cultures: Passionate play. Routledge: New York, NY, 2012.

Taylor, T. L. "Does WoW change everything? How a PvP server, multinational player base, and surveillance mod scene caused me pause," in Games and Culture vol. 1 no. 4, 318-337, 2006.

Tedlock, B.. "Ethnography and ethnographic representation", in N. K. Denzin and Y. S. Lincoln (eds.), Handbook of qualitative research (pp. 455-486). Sage: Thousand Oaks, CA, 2000.

Thelwall, M.. Researching the public web. 12 June 2010. Available at http://eresearch-ethics.org/position/researching-the-public-web/ (accessed Feb. 2012)

Valve. Counter-Strike [PC] Valve Corporation: Bellevue, WA, 1999.

Valve. Team Fortress [PC] Valve Corporation: Bellevue, WA, 1999.

Valve. Left 4 Dead 2 [PC] Valve Corporation: Bellevue, WA, 2009.

Waskul, D. "The role-playing game and the game of role-playing: The ludic self and everyday life," in P. J. Williams, S. Q. Hendricks, and K. W. Winkler (eds.), Gaming as culture: Essays on reality, identity, and experience in fantasy games. McFarland \& Company: North Carolina, 2006.

Zagal, J., M. Mateas, C. Fernandez-Vara, B. Hochhalter and N. Lichti. "Towards an ontological language for game analysis," in Proceedings of the Digital Games Research Association Conference, DiGRA, Vancouver B.C., June 2005. 